Canadian Art Review

\title{
Art et nouvelles technologies : pour un recadrage de la subjectivité humaine par rapport à l'idée de paysage
}

\section{Édith-Anne Pageot}

Volume 35, numéro 1, 2010

Landscape, Cultural Spaces, Ecology

Paysages, espaces culturels, écologie

URI : https://id.erudit.org/iderudit/1066801ar

DOI : https://doi.org/10.7202/1066801ar

\section{Aller au sommaire du numéro}

\section{Éditeur(s)}

UAAC-AAUC (University Art Association of Canada | Association d'art des universités du Canada)

\section{ISSN}

0315-9906 (imprimé)

1918-4778 (numérique)

\section{Découvrir la revue}

Citer cet article

Pageot, É.-A. (2010). Art et nouvelles technologies : pour un recadrage de la subjectivité humaine par rapport à l'idée de paysage. RACAR : Revue d'art canadienne / Canadian Art Review, 35(1), 42-53.

https://doi.org/10.7202/1066801ar

\section{Résumé de l'article}

This article addresses the impact of new media on concepts of landscape. More precisely, it concerns artistic projects using new media that incorporate elements of nature. It shows how these projects participate in an economy of landscape, but at the same time reformulate the very principles associated with the genre. Four projects are presented as case studies: Glenlandia (2005-07) by Susan Collins, Osmose (1995) by Charlotte Davies, Tele-Garden (1995-2004) by Kenneth Goldberg and Joseph Santarromana, and One Tree(s) (1999-) by Natalie Jeremijenko. In essence, these projects deal not with ecology so much as with new spatio-temporal relations of the perceiving subject with nature, relations always mediated by the concept of landscape. As defined by "ecosophy," these spatio-temporal relations are open to exploration and adaptation characterized by continuous change. These changes include distortion, movement, and a form of blindness that weaken the dominant position of vision in human perception of the world. The projects in question overturn the opposition between humanity and nature that had been, from the beginning of the modern era, the basis of landscape, a genre characterized by frontality, alterity, and pure opticality.
Tous droits réservés (C) UAAC-AAUC (University Art Association of Canada | Association d'art des universités du Canada), 2010
Ce document est protégé par la loi sur le droit d'auteur. L'utilisation des services d’Érudit (y compris la reproduction) est assujettie à sa politique d'utilisation que vous pouvez consulter en ligne.

https://apropos.erudit.org/fr/usagers/politique-dutilisation/ 


\title{
Art et nouvelles technologies : Pour un recadrage de la subjectivité humaine par rapport à l’idée de paysage
}

\author{
Édith-Anne Pageot, Université d’Ottawa
}

\begin{abstract}
This article addresses the impact of new media on concepts of landscape. More precisely, it concerns artistic projects using new media that incorporate elements of nature. It shows how these projects participate in an economy of landscape, but at the same time reformulate the very principles associated with the genre. Four projects are presented as case studies: Glenlandia (2005-07) by Susan Collins, Osmose (1995) by Charlotte Davies, Tele-Garden (1995-2004) by Kenneth Goldberg and Joseph Santarromana, and One Tree(s) (I 999-) by Natalie Jeremijenko. In essence, these projects deal not with ecology so much as with new spatio-temporal relations of the perceiving subject with nature, relations always mediated by the concept of landscape. As defined by "ecosophy," these spatio-temporal relations are open to exploration and adaptation characterized by continuous change. These changes include distortion, movement, and a form of blindness that weaken the dominant position of vision in human perception of the world. The projects in question overturn the opposition between humanity and nature that had been, from the beginning of the modern era, the basis of landscape, a genre characterized by frontality, alterity, and pure opticality.
\end{abstract}

$\mathrm{I}_{1}$ est généralement admis que les nouvelles technologies—le numérique et les biotechnologies_engendrent une certaine restructuration de l'économie visuelle ainsi qu'une manière différente de percevoir et d'appréhender le monde ${ }^{1}$. Bien que nous n'ayons pas encore pleinement mesuré les impacts de ces changements, nous pouvons globalement affirmer qu'ils engagent de nouvelles façons d'être ${ }^{2}$ en nous forçant à reconsidérer notre ontologie et notre être-ensemble 3 . Plusieurs artistes se sont emparés de ces nouveaux dispositifs spatio-temporels pour réaliser des projets qui encouragent une réflexion critique sur leur logique même en proposant des configurations existentielles inédites. Peu ou prou, ces projets artistiques contribuent à modifier le paradigme relatif à notre façon de représenter le monde et à notre rapport au temps et à l'espace. Retiendront notre attention les projets intégrant des éléments réels ou virtuels qui réfèrent à la nature; nous pensons par exemple au travail de Stephan Barron, Susan Collins, Luc Courchesne, Charlotte Davies, Kenneth Goldberg et Joseph Santarromana, Natalie Jeremijenko, Christa Sommerer et Laurent Mignonneau. Leurs projets qui sont réalisés pour le web et/ou fondés sur des systèmes numériques, électromécaniques ou biotechnologiques visent à créer des mondes virtuels ou réels qui incorporent des éléments de la flore (arbre, feuille, forêt, jardin) ou de l'atmosphère (eau, nuage, luminosité) et comportent une dimension participative d'importance variable. Cet essai entend soumettre quatre projets aujourd'hui célèbres à la discussion : Glenlandia (2005-07) de Susan Collins, Osmose (1995) de Charlotte Davies, Tele-Garden (1995-2004) de Kenneth Goldberg et Joseph Santarromana et One Tree(s) (1999-) de Natalie Jeremijenko. Loin de prétendre à l'exhaustivité, ces choix offrent néanmoins des exemples significatifs d'œuvres recourant à des éléments naturels réels ou à des " images " 4 de la nature et illustrant différents types d'œuvres médiatiques soit : l'hypermédia, l'œuvre collaborative et relationnelle ainsi que le recours à la cyberception et à la téléprésence 5 .
Nous voulons montrer que ces projets artistiques participent d'une économie du paysage en "reformatant " des références théoriques traditionnelles associées à la notion même du genre, qui est si chère aux arts visuels. Ce faisant, ils opèrent sur un terrain d'intervention qui semble bien être celui de l'histoire de l'art. La révision qu'ils proposent contribue à formuler une réorientation du rapport individu/nature tout en offrant théoriquement des modèles capables de renouveler les dispositifs de production de la subjectivité. Considérés dans cette perspective, ces projets artistiques touchent aussi à l'écologie, entendue comme une " écosophie " au sens de Félix Guattari', et renferment nécessairement une dimension politique. Puisque la capacité de l'art contemporain à bouleverser les conditions de l'existence ou à transformer la société a été sérieusement mise en doute depuis quelques décennies déjà, ce dernier aspect appelle d'emblée une précision. Aujourd'hui, la portée caritative, salvatrice, subversive ou militante de l'art contemporain semble en effet peu convaincante. Certes, plusieurs projets artistiques regroupés sous l'appellation "art écologique " aspirent à une forme de mobilisation militante qui tente de faire échec aux effets d'homogénéisation créés par les médias et aux attitudes de passivité, voire même d'aveulissement, que ceux-ci encouragent. Il s'agit là d'un art à visée politique. Toutefois, aussi louable soitil dans ses intentions, ce type de pratique n'aboutit pas nécessairement à une émancipation collective. C'est aussi le cas d'un " art écologique " dont ont peut douter de la capacité à freiner la pollution, ainsi que Jacques Rancière en fait le constat dans l'introduction de Malaise dans l'esthétiqueך. La "radicalité artistique " est aujourd'hui perçue comme une utopie, résume-t-il, mise en échec par les grands projets totalitaires et l'esthétisation de la marchandise. Le verdict de Dominique Baqué est encore plus sévère quand elle affirme :

L'art contemporain s'avère souvent naï, idéologiquement faible, encore pétri d'illusions humanistes—quand elles ne dérivent pas vers un "caricaturisme " douteux-, clivé entre 
des positions désormais caduques (les néo-avant-gardes) ou des postures relationnelles qui, prônant une convivialité de bon aloi, occultent gravement l'extrême dureté des fractures sociales ${ }^{8}$.

Selon Baqué, le constat d'échec est double : les néo-avantgardes (Haacke, Holzer, Kruger, Orta, Wodiczko, etc.) des années 1980 ne sont pas parvenues à éveiller les consciences aliénées, pas plus que " l'œuvre infra-mince ${ }^{9}$, frisant parfois la " fadeur " voire l' " insignifiance " 10 , des attitudes dites " relationnelles » (Angela Bulloch, Gabriel Orozco, Pierre Joseph, Rirkrit Tiravanija, etc.) des années 1990 n'a su restaurer le lien social. Selon Baqué, la dimension imperceptible de l'infra-mince caractérise l'activité des artistes relationnels et, de fait, invalide leur impact sur la restauration du lien social et sur la notion même de convivialité qui, selon elle, est "forcée ", restreinte à une élite bourgeoise fréquentant les lieux de diffusion de l'art contemporain et incapable de tenir compte des divisions sociales ${ }^{11}$.

L'art contemporain serait-il donc complètement destitué de toute puissance politique? Considérons plutôt, à l'instar de Rancière, que l'art est politique non pas par son message, revendicateur ou dénonciateur, donc non pas par sa portée révolutionnaire, mais plutôt par la manière dont il élabore un certain espace-temps commun distinct des formes ordinaires d'être ensemble. Comme l'explique Rancière, " le propre de l'art est d'opérer un redécoupage de l'espace matériel et symbolique. Et c'est par là que l'art touche à la politique ${ }^{12}$. "C'est donc dans la " re-disposition " des objets communs, " parfois à peine décalée de la vie ordinaire ", dans la création de situations propres à modifier nos regards, dans la manière d'investir le temps que l'art contemporain est politique. Et Rancière d'ajouter :

La politique, en effet, ce n'est pas l'exercice du pouvoir et la lutte pour le pouvoir. C'est la configuration d'un espace spécifique, le découpage d'une sphère particulière d'expérience, d'objets posés comme communs et relevant d'une décision commune, de sujets reconnus capables de désigner ces objets et d'argumenter à leur sujet ${ }^{13}$.

Nous verrons que Glenlandia, Osmose, Tele-Garden et One Tree(s) participent effectivement à une telle re-disposition de l'espace-temps lié à l'idée de " paysage ». Ce dernier point peut certainement être rapproché d'une " écosophie ». Guattari avait d'ailleurs pressenti la portée des nouvelles technologies, et ce dès 1989, lorsqu'il écrivait dans ce texte aujourd'hui consacré : "J'ai la conviction que la question de l'énonciation subjective se posera de plus en plus à mesure que se développeront les machines productrices de signes, d'images, de syntaxe, d'intelligence artificielle ${ }^{14}$.»

Comme l'écrit encore Guattari : «Il n'y aura de réponse véritable à la crise écologique qu’à l'échelle planétaire et à la condi- tion que s'opère une authentique révolution politique, sociale et culturelle réorientant les objectifs de la production des biens matériels et immatériels ${ }^{15}$.» Aussi, remarque-t-il, la seule gestion des problèmes de nuisances industrielles demeure largement insuffisante à l'édification d'une véritable culture de développement durable. Celle-ci dépend non seulement de solutions techniques d'assainissement de lieux contaminés ou de programmes sectoriels de gestion, mais aussi d'un remaniement profond de nos manières de vivre en société touchant l'éthique, la politique et la définition de la subjectivité humaine. Dans cette perspective, Guattari propose de parler d'“ écosophie » pour désigner l'articulation éthico-politique de trois registres de l'écologie, soit : l'environnement, les rapports sociaux et la subjectivité. À propos de celle-ci, le philosophe réclame la reconstruction de «l'ensemble des modalités d'être-en-groupe». Et cela, pas seulement par des interventions " communicationnelles " mais " par des mutations existentielles portant sur l'essence de la subjectivité16.» La mondialisation du capitalisme a entraîné selon lui une trop grande homogénéisation de la conscience, voire même de l'inconscient. Et la normalisation de la conscience humaine entraîne une déresponsabilisation de la gestion de la technique et des sciences menant à des désordres écologiques. À cet égard, la catastrophe de Tchernobyl constitue un cas exemplaire. Ce mouvement de standardisation dû à l'action des multinationales et à une gestion purement technocratique des technologies s'accompagne encore d'une augmentation de la différence entre les pauvres et les riches. Afin de pallier les effets normalisateurs de la mondialisation, Guattari fait l'éloge d'une subjectivité critique, créatrice, innovatrice et libre. Les mutations existentielles et essentielles que réclame le philosophe impliquent de nouveaux " agencements d'énonciation ${ }^{17}$ ", non pas ancrés dans le passé, legs du modèle freudien, mais engagés vers un devenir fondé sur l'innovation et l'adaptation continues, ou en d'autres mots, sur des " ouvertures prospectives 18 " et des " processus de mise à l'être ${ }^{19}$ ".

Il serait naïf de postuler que les nouvelles technologies ont cette capacité de mener à des changements existentiels allant dans le sens d'une écosophie telle que la revendique Guattari, loin s'en faut. Ce nouveau "système des objets " ne s'inscrit pas en rupture avec le passé et répond toujours aux exigences du capitalisme et de l'industrie même s'il s'ajuste à leurs formes les plus contemporaines. D'ailleurs, les innovations technologiques majeures, utilisant notamment la réalité virtuelle, ont d'abord été développées à des fins militaires ${ }^{20}$. Elles ne mènent pas nécessairement non plus à une révolution des modèles traditionnels de conception de l'espace. À ce sujet, rappelons que le dispositif scénique de bon nombre de jeux vidéos s'intègre (parfois jusqu'à s'y confondre) à l'esthétique propre aux vieilles représentations euclidiennes. Bien que le cyberespace ${ }^{21}$ puisse théoriquement convier à des formes de spatialité radicalement 
différentes de celles auxquelles nous a accoutumés la tradition occidentale depuis la Renaissance, nous ne les avons pas encore intériorisées. Anne Cauquelin le souligne :

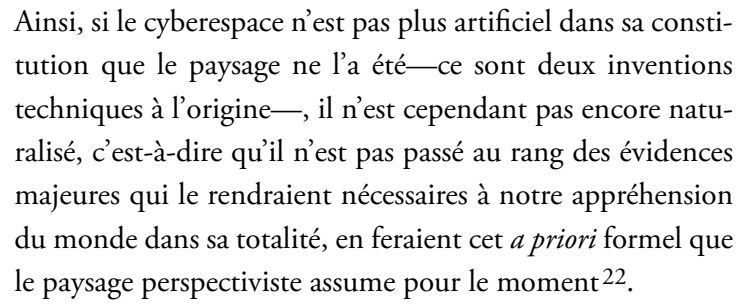

Les projets artistiques dont il est question dans cet essai ménagent quant à eux un espace critique et proposent une nouvelle appréhension, voire une nouvelle définition, de la notion de " paysage ".

\section{Paysage : une notion équivoque}

Comme le remarque avec justesse Anne Cauquelin, le paysage, dans sa forme traditionnelle, continue encore aujourd'hui de constituer un cadre de référence pour notre appréhension du monde. Mais que faut-il comprendre au juste par le mot paysage? Les études récentes en sciences humaines ont eu beau revisiter la notion en tentant de lui inculquer une certaine rigueur ${ }^{23}$, celle-ci demeure encore l'objet de méprises, comme l'ont signalé plusieurs philosophes 24 . L'expression plus récente de " paysage virtuel », constamment galvaudée, vient ajouter à la confusion. Un détour par la terminologie et par la dimension épistémologique qui la sous-tend peut ici s'avérer utile. Par exemple, en anglais, le terme landscape avait originellement une portée administrative et politique et concernait un territoire habité. La brillante étude de Kenneth Olwig montre effectivement que landscape provient du mot allemand landschaft, lequel se référait, au moment de son apparition au IXe siècle, à une communauté organisée de serfs attachée à une terre, frappée de diverses incapacités et assujettie à certaines obligations et redevances. Comme l'explique Olwig : "A Landschaft was more than a place; it expressed the very idea of political representation as manifested in the representative body that stood for a political community ${ }^{25}$.» Son dérivé moderne landscape désigne également une région géographique régie par un ensemble de normes coutumières. Le mot landscape est donc, depuis l'origine, intimement lié à un lieu géographique et, par extension, à la construction d'une identité nationale. Dans ce contexte, la représentation visuelle du paysage contribue à rattacher le territoire à un corps politique. L'analyse incontournable de Simon Schama ${ }^{26}$ portant, entre autres, sur la peinture allemande et sur la forêt hercynienne, montre pour sa part que le paysage est un produit culturel participant à la définition d'un lieu, de sa géographie, de son histoire et de ses récits mémoriels. Aussi,
Schama envisage-t-il le paysage comme un lieu investi par l'homme, le terme anglais " landscape " étant davantage compris comme un écoumène, c'est-à-dire un concept dérivé de la notion de milieu humain pour désigner une " terre habitée $" 27$. Cette archéologie du mot ne lève pas pour autant les ambiguïtés du vocabulaire entourant le terme " paysage " en français. Certes, "pays[age] ", son étymologie en témoigne, signifie toujours une étendue de pays. Toutefois, comme le montre l'étude de Catherine Franceschi 28 portant sur la signification du mot au sein de la tradition latine, en français le "paysage " apparaît d'abord dans le vocabulaire des peintres européens de la Renaissance, dont Ambrogio Lorenzetti (1290-1348) offre un des plus anciens cas de figure. On trouverait pour la première fois le mot " paisage " dans le dictionnaire de Robert Estienne, paru en 1549, où il désigne un mot " commun entre les painctres »; le terme français paysage revêt donc d'entrée de jeu une signification d'ordre pictural, ce qui le distingue des termes landscape et landschaft même s'il renvoie comme ces derniers à une réalité territoriale. En français, le paysage est déjà représentation; il cherche à rendre les beautés et la spécificité d'un site naturel. D'ailleurs, on continue aujourd'hui communément d'associer le «paysage » aux tableaux et aux photographies qui magnifient la splendeur d'un coin de pays.

Dans tous les cas, cependant, le paysage se présente comme une élaboration culturelle qui découle de valeurs d'usage et de valeurs symboliques. Il est le fruit de constructions mentales artificielles naturalisées. Lorsqu'à la Renaissance les peintres s'approprient le paysage, ils l'envisagent à partir d'une expérience optique structurée par l'espace euclidien et fondée sur la base du regard unilatéral, fixe et immobile qu'imposent l'espace perspectif et l'utilisation de la tavoletta. Dès la fin du XVIe siècle, la camera obscura isole l'observateur dans un lieu clos, privé de lumière, et le confine à un certain retrait du monde qui apparaît nécessaire à la régularisation et à la purification du monde extérieur. Ce qui implique une identification du sujet à l'œil omniscient de la caméra. Au modèle euclidien de la perspective linéaire s'attache l'idée que l'œil est un intermédiaire entre la matière et le divin. En tant que représentation d'une réalité purement matérielle, le paysage assujettit la nature à la dominance de l'esprit et impose une mise à distance. Cette conception est cohérente avec certaines idées sur la nature issues de la pensée de Locke. Comme l'explique Arnold Berleant ${ }^{29}$, l'idée de nature en Occident se prête à diverses interprétations qui se rattachent de près ou de loin à l'une ou l'autre de deux positions antagonistes qu'incarnent respectivement John Locke (1632-1704) et Baruch de Spinoza (1632-77). À l'opposé de Spinoza, pour qui la nature est totalement inclusive, Locke comprend cette dernière comme une force vivante, essentiellement extérieure à la civilisation et donc à conquérir. À partir de la Renaissance, le genre pictural " paysage " participe de l'intériorisation d'une telle vision 
des choses, c'est-à-dire de la nature comme cet " autre " contre lequel la culture s'éprouve. Aux avancées de la science optique au XIXe siècle correspond une autre représentation du "paysage ». Comme l'ont montré Jacques Aumont et Jonathan Crary, on comprend dorénavant la vision humaine comme étant relative, bifocale et mobile. Du coup, on constate à quel point la perception s'enracine dans la corporalité 30 . Aussi, le paysage peint devient-il plus incarné et plus dynamique, composé de taches poreuses chez Monet, de points vibrants chez Seurat ou d'un vortex de formes chez Turner. De ce point de vue, le paysage impressionniste semble radicalement différent de celui issu de l'expérience euclidienne. Par contre, dans le contexte de l'industrialisation croissante des villes, le " paysage "—en particulier les vues banlieusardes d'Asnières, de Bougival, de Bois-Colombes ou d'Argenteuil—camoufle les impacts négatifs des industries avoisinantes ${ }^{31}$. Du coup, le paysage continue d'assumer une fonction de repoussoir. La peinture impressionniste maintient un lien obligé entre le paysage et les politiques du territoire et alimente une vision romantique et idyllique de la nature. Or, depuis la fin du XIXe siècle déjà, l'écologie et la biologie modernes ont montré qu'il n'existe pas de rapports tangibles d'extériorité entre l'homme et la nature. Les limites sur lesquelles s'appuierait cette prétendue extériorité sont en effet vagues, poreuses et variables. On situe dorénavant les organismes vivants dans un réseau complexe d'interactions mutuelles comportant des facteurs et des conditions physiques, chimiques, géographiques et culturelles. Comme le dit si bien Arnold Berleant : «Environment is nature experienced, nature lived ${ }^{32}$.» Pourtant, force est de constater que cette conception du monde ne semble pas avoir pénétré pleinement notre langage, ni nos habitudes de vie. Il demeure toujours difficile d'échapper à une conception binaire du monde. Dans ce contexte, le " paysage " continue d'être associé à une mise à distance de la nature, à un acte de contemplation désintéressé et non à un sentiment d'habitation d'un territoire ou mieux encore à un vécu de "nature ", conceptions qui, elles, impliquent un engagement actif. En quoi Glenlandia, Osmose, Tele-Garden et One Tree(s) opèrent-ils des déplacements par rapport à ces modèles ?

\section{Glenlandia}

Le projet Glenlandia33 (2005-07) de Susan Collins s'inscrit dans la continuation de projets antérieurs, notamment Transporting Skies (2002) et, plus récemment, Fenlandia (2004-06), pour lequel Collins avait été invitée par Steven Bode, de la compagnie Film and Video Umbrella, et la Norwich School of Art and Design à explorer le rapport entre le paysage et les innovations technologiques dans la région de Silicon Fen en Angleterre. Pour Glenlandia, Collins a filmé le secteur de Loch Faskally, Perthshire, en Écosse. Loch Faskally est reconnu pour incarner la beauté du paysage écossais dans toute sa quintessence, dans toute son immutabilité. Le site fut pourtant entièrement remodelé par le temps, sa configuration actuelle étant le résultat d'une accumulation de changements dus à la construction des barrages hydroélectriques de Pitlochry entre 1947 et 1950. Pour traduire cette idée de transformation continue du paysage, Collins a installé une webcam à l'extérieur, sur le site des laboratoires de la Fisheries Research Services ; la caméra est demeurée en place pendant deux ans. Du 10 septembre 2005 au 10 septembre 2006, les images de Glenlandia furent diffusées en direct à la Threshold Artspace et elles demeurèrent disponibles sur le web jusqu'en 2007. Les données captées par la webcam étaient transmises à un écran plasma à raison d'un pixel par seconde. À ce rythme, la complétion d'une seule image demandait 76, 800 secondes, soit 21,33 heures 34 . La lenteur de l'archivage provoqua ainsi l'apparition de taches, de scories et d'autres éléments de surprise 35 , autant de contaminations de l'image qui résultaient en fait de mouvements aléatoires et des changements climatiques enregistrés (vols d'oiseaux, déplacements des nuages, fluctuations de luminosité, etc.). La lenteur de la transmission des mouvements a donc eu pour effet d'amenuiser la lisibilité de l'image tout en révélant la complexité des interactions de l'environnement et en rendant tangibles des données environnementales habituellement impalpables. La captation d'éléments dynamiques et météorologiques sur laquelle repose ce type de dispositif s'inscrit dans le prolongement de la grande tradition du paysage atmosphérique : on pense aux paysagistes modernes anglais de Ruskin, à Prout, Fielding, Harding et Constable jusqu'à Turner. Le goût des modernes anglais pour les éléments aériens témoigne assurément d'une vision romantique du paysage, de l'intérêt pour une nature incommensurable, affranchie de la domination humaine et exprimant un désir de liberté. Dans le contexte plus large de l'histoire du paysage de la Renaissance aux modernes, ces éléments jouent un rôle central sur l'imagination, révélant et dissimulant tout à la fois l'irreprésentable. Comme l'explique Hubert Damish, le nuage et le brouillard incarnent le mystère et l'infinité des choses 36 . Les représentations picturales du nuage iront parfois jusqu’à revêtir une valeur d'objet hallucinatoire où le motif fonctionne comme un fétiche investi d'un désir d'expérience spirituelle. Il en va ainsi des quatre panneaux mythologiques commandés par Frédéric de Gonzague au Corrège et consacrés aux amours de Jupiter dans lesquels ce dernier apparait, entre autres, sous forme de nuées. Damish écrit à propos de ce corpus: «Si le nuage est l'un des signes sur lesquels s'ordonne le désir mystique d'union avec Dieu, il reste qu'il se donne dans ce tableau pour un objet hallucinatoire, lié comme tel à une expérience " ascensionnelle " fondamentale, mais rien de moins que spirituelle : celle d'une tension érotique et de sa résolution sur le mode de l'effusion onirique ${ }^{37}$.» 
Du Corrège, en passant par Léonard pour arriver aux modernes anglais, le paysage se sécularise, mais il reste que chacun reconnaît dans ses éléments aériens les figures de son désir. Les projets de Collins portent, eux aussi, les signes et les fantasmes de sa culture, en l'occurrence d'une culture contemporaine pénétrée d'expériences de transition, de changement continuel et de mobilité. Au-delà d'une saisie de la nature confinée à la géométrie euclidienne, le paysage se déploie et s'appréhende dans une dimension temporelle. Le dispositif de Collins expose la capacité limitée du système cognitif humain à appréhender la nature dans sa dimension essentiellement évolutive et mue par des lois thermodynamiques. Cette nouvelle conjoncture implique un autre régime de sensibilité qui tient compte du mouvement et du temps ainsi que de l'expérience que l'on en fait.

\section{One Tree(s)}

Les préoccupations au cœur de One Tree(s) recoupent celles qui motivent d'autres pratiques multidisciplinaires contemporaines appelées eco-visualization ${ }^{38}$, en ce que ce projet vise à divulguer et à vulgariser des données environnementales spécialisées et généralement peu accessibles ou mal comprises par le grand public. Comme l'explique Jeremijenko sur le site web du projet, One Tree(s) satisfait à une volonté de démocratiser l'accès à la connaissance scientifique en faisant vivre à une communauté de non-experts l'importance du déterminisme environnemental sur le " fait naturel ». Mais par ailleurs, One Tree(s) entend mettre en perspective, en les complexifiant, les enjeux politiques et sociaux du réchauffement climatique, des modifications de la qualité de l'air et de l'utilisation d'organismes génétiquement modifiés en révélant et en défiant les mécanismes de pouvoir de l'information scientifique. D'entrée de jeu l'artiste affirme :

The OneTrees instrument however, captures a different way of conceiving of information with respect to the trees. Not information packets produced as simplified data product to be passively consumed, rather, it privileges a conception of information that requires interpretation. It demonstrates that this complex multi-parameter phenomena of growth can be understood in many way, can sustain many interpretations and can be 'read' from the material phenomena itself, not as a pre-interpreted digested data packet, not delivered by an expert, not wrapped in the incontestable authority of science 39 .

Le projet One Tree(s) comporte trois volets : la plantation réelle d'arbres clonés (One Tree(s) proprement dit), un logiciel de vie artificielle (A-life Trees) et un virus électronique (Stump). A-life Trees (artificial life trees) est un logiciel qui permet à l'utilisateur de simuler la croissance d'un arbre virtuel sur son propre écran d'ordinateur. L'arbre virtuel étant modélisé d'après le clone bio- logique, il s'agit en quelque sorte d'un clone algorithmique. La croissance du clone virtuel varie selon le taux de dioxyde de carbone $\left(\mathrm{CO}_{2}\right)$ contenu dans l'air ambiant de la maison ou du bureau de l'utilisateur, enregistré par le logiciel. Stump est un virus électronique qui comptabilise le nombre de feuilles de papier consommées par l'imprimante de l'utilisateur. Lorsque la quantité de ces feuilles correspond à la quantité moyenne de fibres contenues dans un arbre, l'appareil imprime automatiquement une coupe de souche d'arbre. Le troisième volet de One Tree(s) consiste dans la plantation d'arbres biologiques clonés. Il est l'aboutissement d'une expérience commencée en 1999 dans les laboratoires de la Modesto's Burchell Nursery où des clones furent cultivés à partir du code génétique du Paradox Vlach, une variété spécifique de noyer ${ }^{40}$. Malgré des conditions de culture identiques et stériles, les boutures obtenues par micropropagation ont rapidement montré des différences notables de taille et de structure. Ce sont justement les propriétés physiques uniques de chaque arbre qu'ont voulu mettre en perspective les expositions des clones intitulées respectivement Ecotopias (tenue au Yerba Buena Center for the Arts à San Francisco du 14 novembre 1998 au 3 janvier 1999) et Paradise Now: Picturing the Genetic Revolution (une exposition itinérante montrée d'abord à la Exit Art à New York en 2000) ${ }^{41}$.

En 2003, Jeremijenko obtient l'autorisation de planter par paires les arbres clonés dans la péninsule de San Francisco Bay, une région qui accuse des écarts considérables de niveaux de vie (entre les secteurs est et ouest de Palo Alto, notamment) et qui loge un nombre important de sites d'enfouissement de déchets toxiques. Pour effectuer les travaux de plantation, Jeremijenko recourt à des équipes bénévoles, des membres du Pond and Friends of the Urban Forest. Parmi les endroits retenus pour faire croître les clones, on trouve entre autres: le Golden Gate Park, des écoles du district de San Francisco, le Yerba Beuna Performing Arts Center, le Union Square, des escaliers publics et quelques 220 bordures de trottoirs en milieux résidentiels. Il va sans dire que la qualité des sols, de l'air et la quantité d'espaces verts environnants rendent certains sites plus propices que d'autres à la croissance du clone et altèrent l'aspect des arbres en modifiant, par exemple, le branchage ou le nombre de feuilles. La qualité des différents microclimats choisis pour la plantation des Paradox Vlach varie donc également en fonction de la valeur sociale qu'on leur attribue. Les espaces verts en milieu urbain sont en effet de puissants symboles de richesse et de pouvoir; le défrichement déréglé des quartiers défavorisés en témoigne par la négative. Aussi les microclimats des secteurs les plus cossus s'avèrent-ils plus fertiles à la croissance des clones que ceux des secteurs pauvres.

One Tree(s) non seulement met à jour les visions réductrices et la confusion qui entourent le phénomène complexe de croissance issu du clonage, mais révèle aussi les écarts sociaux qui 
façonnent le paysage urbain. De plus, il appelle un engagement citoyen actif. L'entretien des arbres entourant un édifice dans la baie de San Francisco étant légalement imparti à son propriétaire, la présence des clones impose sa participation tout en le conviant à une réflexion sur le rapport entre la pollution, les inégalités sociales et les stéréotypes liés au clonage. Et Jeremijenko de dire :

In the public sphere, genetics is often reduced to 'finding the gene for...(fill in the blank)', misrepresenting the complex interactions with environmental influences. The swelling cultural debate that contrasts genetic determinism and environmental influence has consequences for understanding our own agency in the world, be it predetermined by genetic inevitability or constructed by our actions and environment. The OneTrees project is a forum for public involvement in this debate, a shared experience with actual material consequences 42 .

Le travail de Jeremijenko vise donc à révéler la rhétorique simpliste et les déformations déterministes qui entourent le clonage. Sont questionnés, voire déconstruits, les présupposés de standardisation ainsi que les désirs de rectitude et d'épuration qui sous-tendent les mécanismes de contrôle (pouvant aller jusqu'au rejet) de certains caractères de la flore jugés indésirables en milieu urbain. C'est d'ailleurs sur la base de ces exigences que le Paradox fut longtemps considéré comme un arbre " urbain " par excellence ne produisant pas de fruits et peu de pollen, lesquels posent problème aux idéologies citadines de pureté et d'asepsie. Les noix qui tombent au sol et le pollen potentiellement allergène échappent en effet au contrôle et, conséquemment, perturbent l'ordre de la ville. Les clones biologiques de One Tree(s)—qui résultent pourtant d'opérations scientifiques " réputées " exercer un contrôle rigoureux de la génétiquen'échappent pas à un certain désordre. La croissance, somme toute déréglementée, du Paradox Vlach cloné vient signaler le caractère utopique et fantasmatique des impératifs d'asepsie, de rectitude et de régulation qui sont à l'origine de nombreux projets d'aménagements paysagers urbains en Occident depuis le début de l'ère moderne. À cet égard, les arbres virtuels et réels de One Tree(s), dont la croissance est imprégnée des effets de l'environnement, personnifient la ligne « courbe ", menaçante, qu'un Le Corbusier rêvait de répudier : " La circulation exige la droite. La droite est saine aussi à l'âme des villes. La courbe est ruineuse, difficile et dangereuse; elle paralyse ${ }^{43}$.» En ce sens, les clones biologiques et algorithmiques de One Tree (s) court-circuitent les idéologies environnementales qui sous-tendent l'aménagement des grands centres urbains nord-américains et figurent, bien paradoxalement ${ }^{44}$, la peur du retour à l'état sauvage. Pour reprendre les mots de Julia Kristeva intéressée par la figure de l'abject, ils sont une « immixtion de l'organique dans le social »45.
On est loin ici d'une sociabilité superficielle ou nivelée ; on se situe davantage dans la perspective d'une "sculpture sociale " à la Beuys, d'un travail actif qui donne à penser, qui incarne les angoisses citadines tout en contribuant à créer un espace public de débats. D'ailleurs, One Tree(s) apparaît clairement comme une réactualisation de l'intervention que Joseph Beuys a intitulée 7000 Eichen (7000 chênes, Documenta 7, 1982). One Tree(s) adapte la notion beuysienne d'œuvre d'art totale et l'étend à des matériaux invisibles, incluant la pensée qui donne forme au monde. À l'instar du projet de Beuys, One Tree(s) offre un espace public d'engagement concret aux utilisateurs, mais il le double d'un espace virtuel privé (Stump et A-life Trees), une micro-politique en quelque sorte, qui accueille les informations et les traite à nouveau selon sa propre logique. One Tree(s) propose ainsi une jointure entre deux espaces (privé et public), deux dynamiques (individuelle et collective) et deux réalités (clonée et simulée) et intègre une réflexion sur les pratiques et sur leurs conditions de réalisation.

\section{Tele-Garden}

Le célèbre projet Tele-Garden (1995-2004), conçu par Kenneth Goldberg et Joe Santarromana ${ }^{46}$, est également une œuvre de type "participatif " impliquant, dans ce cas-ci, une collaboration collective aidée de la télérobotique. On se rappellera que Tele-Garden consistait en un jardin communautaire miniature, géré à distance par une communauté internationale d'étrangers au moyen d'un bras robotique. Le concept fut développé à la University of Southern California et mis en ligne en juin 1995. En septembre 1996, le jardin fut déménagé dans les locaux du ARS Electronica Museum à Linz en Autriche. La connexion au jardin via Internet est restée active jusqu'en août 2004. L'accès au jardin se voulait inclusif et non discriminatoire. Par conséquent, quiconque voulait participer à ce projet n'avait qu'à suivre une procédure simple d'inscription à partir d'Internet. Les opérations concrètes de creusage, de plantation, d'arrosage et d'entretien du jardin lilliputien étaient contrôlées à distance par les internautes au moyen du bras robotique. Ce dernier, muni d'une caméra, permettait au participant/regardeur de réaliser et de vérifier l'exécution des commandes à partir d'Internet. Dès la première année de sa mise sur pied, Tele-Garden a attiré la participation de quelque 9000 internautes. Depuis ce temps, environ 100000 personnes ont visité le site dont 10000 étaient membres et ont activement participé à ce projet. Tele-Garden a donc engendré la formation d'un véritable village virtuel où les participants pouvaient échanger leurs coordonnées ${ }^{47}$. Ce dispositif relève assurément de ce qu'Edmond Couchot appelle une esthétique de la commutation ; les échanges en ligne, en temps réel, établissent en effet une "liaison de tous vers tous", " tout de suite " 48 . Ici, le message ne préexiste plus à sa transmission mais 
se constitue pendant le dialogue. Comme le défend Edmond Couchot, en raison de son caractère dialogique et de sa rapidité de transition, ce type de dispositif relève non plus d'une esthétique de la communication (de un vers tous), mais plutôt d'une esthétique de la "commutation » en lien avec les approches connexionnistes 49 .

Oliver Grau explique l'enthousiasme que suscitent de tels projets de téléprésence par la persistance d'un désir de transcendance et d'omniprésence, voire d'ubiquité, tel qu'on le retrouve dans plusieurs mythes et religions: "Robotics, telecommunications, and virtual reality feed into history of the idea of telepresence-three areas that from their inception have featured repeatedly interpretations of the given technical stage of development charged with mythological/magical or religious overtones ${ }^{50}$.» Il va sans dire, en effet, que l'histoire recèle plusieurs autres dispositifs, bien antérieurs à la télérobotique, qui matérialisent cette aspiration à transcender la prémisse matérialiste. Le panorama en est déjà curieusement un cas de figure. Il fut dérivé de l'invention de Robert Barker, appelée « la nature à coup d'œil " (1787), qui consistait à déployer une toile circulaire entièrement peinte sur laquelle étaient représentées des vues topographiques d'une ville patrimoniale, d'un paysage sublime ou de lieux exotiques. Le spectateur entrait dans une rotonde et occupait une balustrade d'où il pouvait littéralement embrasser le " paysage ». Dans le contexte colonial du XIXe siècle, le panorama a servi à faire vivre une expérience d'immersion à un public de touristes bourgeois en mal d'exotisme ${ }^{51}$. En comparaison, la télérobotique démocratise (puisqu'elle la rend accessible à tous ceux qui ont accès à Internet) l'expérience du panorama. Tele-Garden généralise ainsi le partage de l'espace commun, du temps et des activités liées au jardin et, en ce sens, redistribue ce que Rancière nomme le "partage du sensible ". Cependant, il faut bien se rendre à l'évidence que, dans l'état actuel des choses, la communication réticulaire universelle souhaitée par un projet tel que Tele-Garden reste souvent la prérogative d'une élite d'initiés liée au monde de l'art. Il n'en demeure pas moins que ce type d'oeuvre radicalise l'expérience du panorama. Alors que ce dernier ne parvenait pas complètement à évoquer un sentiment fort de présence ou d'immersion ${ }^{52}$, Tele-Garden élimine la distance et multiplie les possibilités d'interventions simultanées.

En principe, ce type de jardin, dont la survie dépend de la robotique, peut transformer profondément notre compréhension de l'espace ainsi que l'expérience que nous en faisons. Il abolit la distance entre l'environnement physique de l'observateur et celui du jardin, une condition qui s'inscrit en porte-àfaux avec une tradition esthétique du paysage fondée sur une distance obligée entre le sujet et l'objet. Parce que l'interface, ici la souris d'ordinateur, n'interrompt pas les processus de calcul, le dialogue a lieu en temps réel. Échappant au désir de péren-

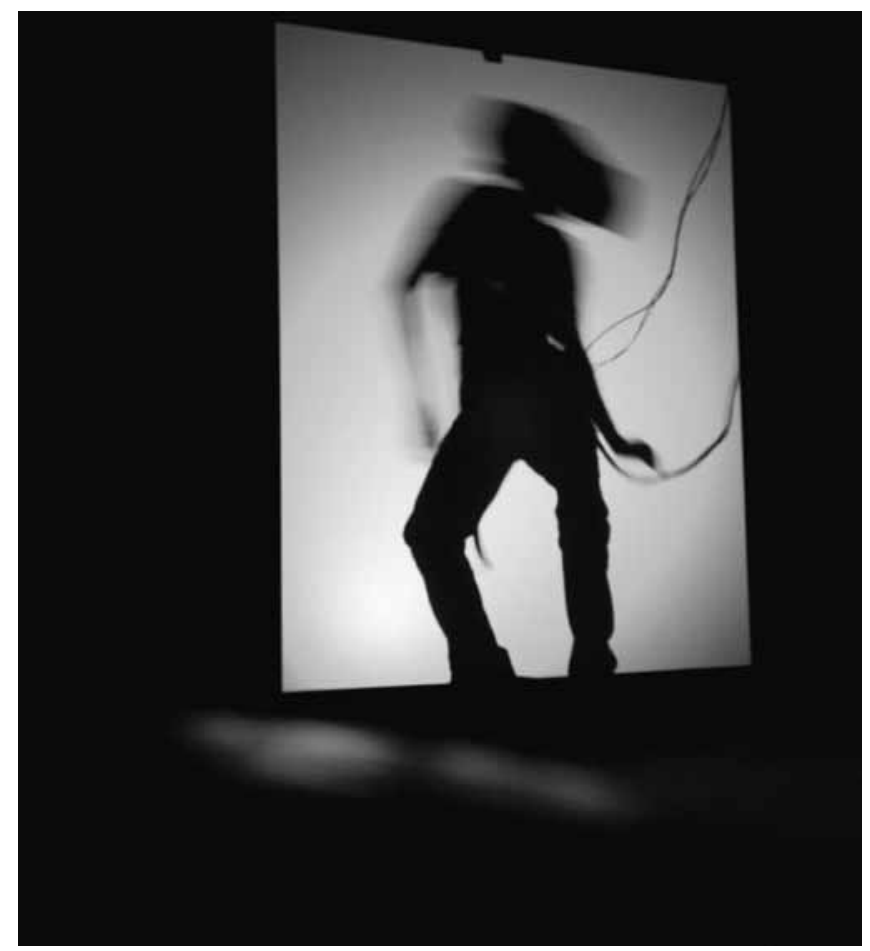

Figure I. Char Davies, Immersant as seen through shadow screen during immersive performance of virtual reality environment Osmose, 1995 (C) Char Davies 2010).

nité-et du coup aux exigences de conservation que cela impose-l'œuvre est " soumise aux contingences et aux aléas de la prise rapide, du live 53 ». La télérobotique s'enracine donc dans une temporalité accélérée et simultanée où la distance ne semble plus avoir d'importance. Or l'esthétique, de Kant à Adorno, a rarement fait l'économie d'une telle distance. Cauquelin disait justement à ce propos : "Nous ne pouvons voir un paysage de l'intérieur, en étant "dedans ». Il nous faut être en dehors ${ }^{54}$.» La psychologie et la psychanalyse modernes n'ont fait que renforcer cette idée en posant le processus de distanciation entre le moi et l'objet comme un élément essentiel à la formation de la subjectivité et de l'autonomie individuelle. Tele-Garden abolit cette distance structurante. Tout comme d'autres projets de Goldberg55, le jardin visait justement à engendrer un questionnement sur les fondements épistémologiques de la télécommunication. Tele-Garden se voulait à cet égard une enquête " télépistémologique ${ }^{56}$ ", questionnant l'étude des formes et des structures de la connaissance transmise et acquise à distance, en tenant compte des leurres possibles que cela implique. Il s'agissait de réfléchir au caractère bipolaire de la télérobotique, à la fois connexion d'interlocuteurs séparés par de grandes distances physiques et «dysconnexion » engendrée par les fraudes et par le faux « live ». On peut, en effet, être sceptique quant à l'authenticité de l'in- 
formation médiatisée par la robotique. D’ailleurs, plusieurs internautes en sont venus à douter de l'existence physique dudit jardin (sans que, curieusement, cela compromette leur intérêt à y participer).

Qu'en est-il de la thématique du jardin lorsqu'elle est envisagée dans son rapport à l'utilisateur ? Le jardin est par définition une étendue de terrain strictement délimitée par des murs ou des clôtures ; il constitue une nature maîtrisée et rendue ouvertement accessible, propice à la manipulation. Mais le jardin est aussi cet espace abstrait, métaphoriquement impénétrable (le jardin d'Eden), qui invite à la méditation. Dans cette perspective, Tele-Garden est une " nature » mise en abîme et paradoxale, doublement clôturée, enserrée par le jardin lui-même et, de surcroît, gardée par le robot, à la fois physiquement inatteignable et complètement disponible depuis les quatre coins du monde. Un paysage délocalisé, qui éveille une réflexion et suscite un doute quant aux effets de la distance sur notre capacité à connaittre et à découvrir le monde sensible. L'oeuvre interroge assurément ce "délire de proximité », que Jacques Rancière identifie comme le " mythe officiel contemporain " 57 , cette " fausse " proximité qui annihile le lointain ou qui nous transporte dans un ailleurs inhabituel, là où se dévoile le paysage comme paysage.

\section{Osmose}

Célébrée internationalement, l'oeuvre Osmose (1995) de la Canadienne Char Davies 58 propose une expérience d'immersion dans la réalité virtuelle. Inspiré de moments passés dans la solitude et la tranquillité des territoires ruraux du sud du Québec, où l'artiste possède une propriété 59 , son dispositif a été conçu dans le but de faire vivre à l'utilisateur une expérience cinétique d'immersion à travers laquelle ce dernier est invité à entrer en symbiose, d'où le titre Osmose, avec une nature simulée. Pour faire l'expérience d'osmose, l'utilisateur est isolé dans une petite pièce où il enfile un casque de vision stéréoscopique, un appareil d'écoute et une veste pourvue de senseurs qui lui permettent d'accéder au "paysage " virtuel conçu par Davies. Les mouvements corporels volontaires et involontaires déterminent les déplacements de l'utilisateur dans l'espace virtuel. La structure spatiale du paysage virtuel d'Osmose intègre divers éléments visuels et sonores : une clairière, une forêt, un étang, des nuages, des feuilles, un univers sous-marin, un abysse, mais aussi une grille, du texte et des codes, ainsi que des échantillons de voix humaines, féminines et masculines. Les éléments sonores sont ici conçus pour faire écho aux éléments visuels. La respiration de l'utilisateur engendre des déplacements virtuels verticaux (allant du code au texte en passant par un espace sous-terrain, une clairière et des nuages) dont la portée est proportionnelle à l'intensité de l'inspiration et de l'expiration. Les mouvements du corps produisent, quant à eux, des déplacements virtuels horizontaux

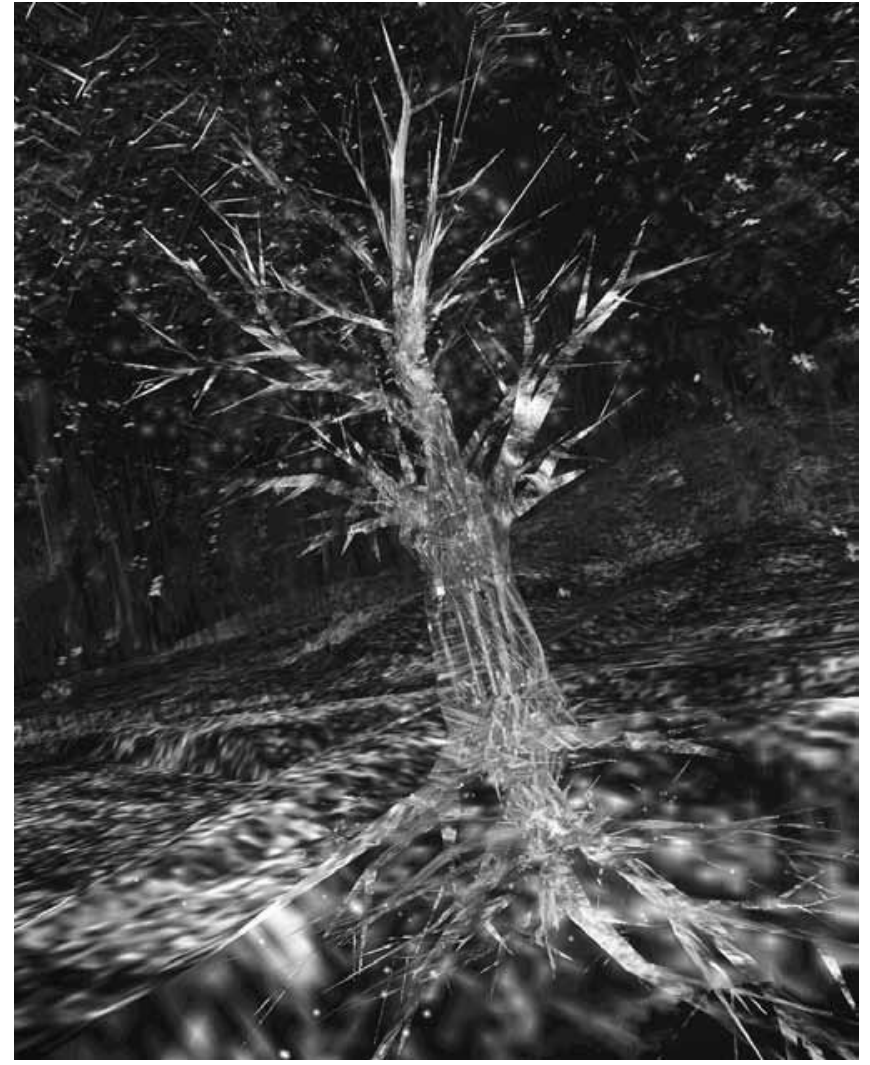

Figure 2. Char Davies, Vertical Tree, Osmose, 1995. Digital still image captured during immersive performance of virtual reality environment Osmose (CChar Davies 2010).

entre une forêt et une clairière virtuelles. Davies opte pour des images élastiques et semi-transparentes, de telle sorte que les limites entre les divers éléments qui constituent le paysage virtuel demeurent floues et baignent dans la lumière. La fluidité de l'espace et des formes évoque une membrane poreuse. Un peu à la manière de ce que saisirait un regard myope (Davies souffrant elle-même sévèrement de cette affliction), l'espace se constitue en volumes ambigus, contrevenant aux formes définies et aux contours tranchants qui sont ceux de l'illusionnisme et du réalisme photographique. Le dispositif d'exposition prévoit également une pièce munie de deux écrans lumineux, qui est ouverte aux autres visiteurs plongés dans une semi-obscurité. La vidéo stéréoscopique du monde 3D dont l'utilisateur fait l'expérience est alors projetée en temps réel sur l'un des écrans. Le deuxième écran projette la silhouette de l'utilisateur et reproduit, un peu comme une ombre, les mouvements de ce dernier (fig. 1).

L'utilisateur est donc convié à "voyager " dans un univers où se déploie un paysage archétypal (figs. 2,3). Comme l'explique Davies, l'intention qui sous-tend l'œuvre est de créer une expérience d'ordre mythique, une quête de réconciliation: 


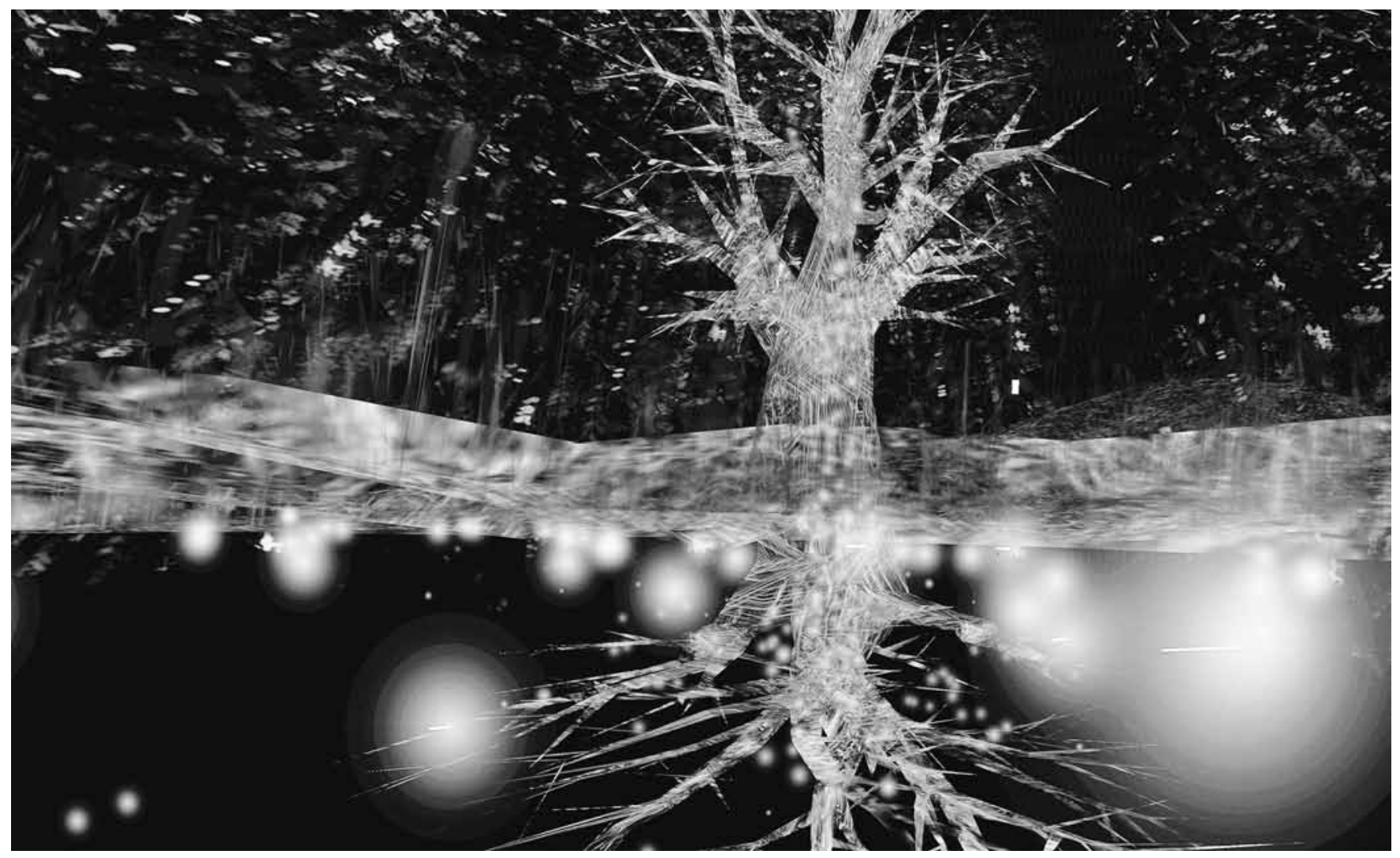

Figure 3. Char Davies, Tree Pond, Osmose, 1995. Digital still image captured during immersive performance of virtual reality environment Osmose (CChar Davies 20l0).

In Osmose, I set out to create a work which not only communicates my own particular vision of the world, but which demonsrates the medium's potential to enable us to experience our place in the world afresh, or to paraphrase Bachelard, to change space in order to change our Nature. [...] In this context, Osmose seeks to re-sensitise-reconnecting mind, body and world 60 .

Délaissant la posture cartésienne, Osmose arrime en quelque sorte les pulsations du corps à une nature archétypale simulée. Directement branchées sur les fonctions vitales de l'utilisateur, les interfaces pénètrent ce paysage de manière tactile ${ }^{61}$. On parlera tantôt de "nouvelles sensorialités ", tantôt de " métasensibilité " pour désigner cet univers intellectuel converti aux désirs du sensible. Louise Poissant, en les situant aux " contours de l'expérience esthétique ", parle en effet de nouvelles sensorialités 62 pour désigner l'amplification des capacités multisensorielles du corps qu'induisent de telles interfaces. Alain-Renaud Alain parle, quant à lui, de métasensibilité63 pour désigner l'arrimage entre l'intelligibilité et le corporel. Lorsqu'il s'agit de caractériser les modalités du toucher dans la réalité virtuelle, Laura Marks utilise, pour sa part, le concept d'une vision tactile (" tactile, or haptic visuality ")64. Contrairement au trompe-l'œil fondé sur une mise à distance obligée, l'accès à la réalité virtuelle repose sur cette appropriation du " paysage " par le corps au moyen d'interfaces sophistiquées. Ce qui fait dire à Derrick de Kerckhove que le 3D « rétablit l'expérience tactile dans une forme visuelle ${ }^{65}$ ». Osmose offre donc des alternatives à nos modes habituels d'appréhension de l'image et, paradoxalement, de la nature en ramenant l'expérience tactile et auditive dans l'expérience visuelle.

\section{Conclusion}

Le numérique, la biotechnologie, la réalité virtuelle et la télérobotique questionnent à différents degrés le rapport du sujet au corps et au temps en inventant une nouvelle conception de l'image que l'on comprend dorénavant comme un espace poly-sensoriel et une expérience interactive possédant une dimension temporelle. Ces dispositifs font exister d'autres possibles et forcent des changements dans nos habitudes perceptives. De toute évidence, et de façon générale, il est clair que ces nouvelles technologies mettent en place de nouveaux agencements 
d'énonciation de la subjectivité. Glenlendia, One Tree(s), TeleGarden, Osmose interrogent à la fois le dispositif technologique et le paysage comme " invention technique ». En dépit de l'activisme de Natalie Jeremijenko, la portée réelle de ces projets n'épouse pas celle d'un art écologique dont le but premier serait d'éveiller les consciences, de modifier le cours de l'histoire ou de freiner la pollution. Ces ouvres travaillent à un autre niveau, sur le dispositif même du rapport spatio-temporel que le sujet percevant entretient avec la nature, rapport médiatisé par le concept de paysage. Ce rapport est ouvert à la prospection et à l'adaptation telles que les réclame l'écosophie et se caractérise par des changements continus qui incluent la distorsionainsi qu'une forme de "délire " de proximité (Collins, Davies, Goldberg, Jeremijenko), la circulation (Collins, Davies, Goldberg), l'intertextualité et la mutation (Jeremijenko, Goldberg), mais aussi une forme de cécité (Davies, Collins, Goldberg) abrogeant la position déterminante de la vision dans l'appréhension du monde sensible. Par conséquent, ces projets artistiques révoquent les oppositions traditionnelles qui, depuis l'ère moderne, ont fondé le " paysage " sur un principe d'extériorité entre l'homme et la nature, ce type de paysage, nécessairement confiné à la frontalité, à l'altérité et à une pure opticalité qui, pour Philippe Poullaouec-Gonidec, "muséifie le territoire "66. Comme le remarque Anne Cauquelin, on assiste aujourd'hui à un «basculement de la philosophie du goût et de la contemplation désintéressée à une philosophie de l'action ${ }^{67}$.»

À l'instar d'autres projets ayant recours aux nouvelles technologies 68, Glenlandia, One Tree(s), Tele-Garden et Osmose mettent en évidence la capacité du dispositif numérique en général et, plus particulièrement, de la télérobotique, de la réalité virtuelle et des biotechnologies, à remettre en cause les vieux dualismes nature/culture, intérieur/extérieur, corps/esprit, intelligibilité/sensibilité. Ces propositions artistiques relativisent toutes les catégories binaires et échappent ainsi au rabâchage des principes théoriques qui ont guidé le rapport de l'homme à la nature, à travers ses représentations en paysages. Glenlandia présuppose l'inscription du " paysage » dans un environnement vivant et mobile, où tout est en état de changement et où tout découle de réactions chimiques, de fermentation, de dégradation, etc. L'espace devient inséparable du temps, comme le veulent les théories de la relativité, restreinte (1905) et générale (1915), d'Einstein. A-life Tree envisage la « nature " à partir de la composition chimique de l'air ambiant. One Tree(s) opère une ré-appropriation de l'information scientifique transmise par les médias et réalise donc implicitement une critique des mécanismes de pouvoir qui y sont à l'oeuvre. Mais encore, le projet ménage un espace public d'intervention qui s'inscrit dans le temps et la durée. One Tree(s) se distingue clairement d'une esthétique relationnelle courtoise, pacifiée et aseptisée. La dissymétrie des clones témoigne des fractures sociales et de la contamination environnementale. Osmose propose une subjectivité assistée par ordinateur qui vise à communiquer une expérience sensible et incarnée du " paysage ", inscrivant ce dernier à l'intérieur de soi. Tele-Garden, tout comme Osmose, abolit la distance structurante $\mathrm{du}$ sujet et, ce faisant, insinue un doute face aux fondements épistémologiques et idéologiques du " paysage ".

Dans tous les cas, ces opérations de reformatage du paysage opèrent une redistribution des rapports entre le corps, l'espace et le temps à travers des éléments de la nature simulés ou réels. Il s'agit là d'un changement important de paradigme. Avec la camera obscura, le paysage était fondé sur une représentation statique de l'espace et du territoire. Au XIXe siècle, il devient incarné dans un corps et, surtout, dans un appareil de vision perçu comme en mouvement. Les projets artistiques contemporains qui recourent aux nouvelles technologies envisagent la construction de " paysages " dans leur relation avec le temps, en tenant compte non seulement du corps et de l'appareil de vision humain, un appareil mouvant et bifocal, mais aussi de l'objet lui-même, de la nature vécue dans son rapport au temps. Celle-ci s'expérimente à travers des réseaux décentrés et se définit davantage en termes de relations et de comportements qu'en territoires géographiquement, politiquement et économiquement circonscrits. Le " paysage " s'en trouve déterritorialisé et, du coup, libéré de son ancrage dans un topos. Sans bornes ni contours, il est irréductible à sa seule dimension géographique. Dans une perspective heuristique, ces projets ont certainement le mérite de contribuer à une connaissance plus approfondie de notre rapport au monde ; pour reprendre les mots d'Anne Cauquelin, ils agissent comme une "interface "69 entre le monde numérique et le monde réel. Mais encore, d'une manière un peu surprenante, ils contribuent à recadrer la subjectivité par rapport à l'idée de nature et à envisager le paysage comme une « nature vécue » qui atteint l'homme dans son être-ensemble.

\section{Notes}

1 Lev Manovich, The Language of New Media, Cambridge, Mass., London, UK, MIT Press, 2000.

2 Roy Ascott est l'un des premiers à avoir tenté, et ce dès 1966, de saisir théoriquement les bouleversements qu'entrainent les technologies de la télécommunication sur la formation de la subjectivité. Voir entre autres : Roy Ascott, « Behaviorist Art and the Cybernetic Vision ", Cybernetica, vol. 9, no. 3, 1966, p. 247-64 ; et plus récemment Roy Ascott (éd.), Reframing Consciousness : Art, Mind and Technology, Plymbridge, Intellect, 1999.

3 Au sujet des conséquences du virtuel sur l'ontologie, on peut consulter : Philippe Quéau, La Planète des esprits. Pour une politique du cyberespace, Paris, Odile Jacob, 2002.

4 Le terme " image " est employé ici avec beaucoup de réserves, car on peut se demander s'il convient encore de parler d' « images » à 
propos de dispositifs qui impliquent une immersion de l'utilisateur.

5 Annik Bureaud, "Pour une typologie de la création sur Internet ", à consulter sur le site de l'observatoire Leonardo des arts, des sciences et des technologies (http://www.olats.org).

6 Félix Guattari, Les trois écologies, Paris, Galilée, 1989.

7 Jacques Rancière, Malaise dans l'esthétique, Paris, Galilée, 2004, p. 9-10 et 31 .

8 Dominique Baqué, Pour un nouvel art politique. De l'art contemporain au documentaire, Paris, Champs, Flammarion, 2004, p. 32.

9 Ibid., p. 150. On se rappellera que Marcel Duchamp avait eu recours au néologisme « infra-mince » pour désigner une catégorie de choses infimes, une limite ou un espace interstitiel, souvent inaperçues ou imperceptibles, comme le bruit que fait par exemple un pantalon de velours lorsqu'on bouge.

10 Ibid., p. 151.

11 Ibid., p. 157.

12 Jacques Rancière, op. cit., p. 37.

13 Ibid.

14 Félix Guattari, Les trois écologies, Paris, Galilée, 1989, p. 14

15 Ibid., p. 31.

16 Ibid., p. 22.

17 Ibid., p. 25.

18 Ibid., p. 30.

19 Ibid., p. 36.

20 À ce sujet voir, entre autres, Olivier Grau, Virtual Art. From Illusion to Immersion, Cambridge, Mass., London, UK, MIT Press, 2003, p. $169-73$.

21 Le terme cyberespace a été introduit en 1984 par le Canadien William Gibson dans son ouvrage de science-fiction Neuromancer. Il désignait alors une hallucination consensuelle quotidiennement expérimentée par des milliards d'opérateurs. Le dictionnaire des arts médiatiques de Louise Poissant le définit ainsi : "Totalité formée par la circulation continue d'informations, depuis les processeurs internes des ordinateurs jusqu'aux réseaux planétaires de télécommunications. » Louise Poissant (dir.), Dictionnaire des arts médiatiques, collection esthétique, Presse de l'Université du Québec, Sainte-Foy, 1997, p. 82.

22 Anne Cauquelin, Le Site et le paysage, Paris, PUF (2002), 2007, p. 194

23 Entre autres: John Barrell, The Idea of Landscape and the Sense of Place, Cambridge, Cambridge University Press, 1986 ; William Cronon, Uncommon Ground: Towards Reinventing Nature, New York, W.W. Norton, 1995 ; W.J.T. Mitchell (dir.), Landscape and Power, Chicago, Chicago University Press, 1994 ; Kenneth Robert Olwig, Landscape, Nature, and the Body Politic: From Britain's Renaissance to America's New World, Madison, The University of Wisconsin Press, 2002 ; Simon Schama, Landscape and Memory, New York, Alfred A. Knopf, 1995 ; Greg Thomas, Art and Ecology in Nineteenth Century France. The Landscapes of Theodore Rousseau, Princeton, New Jersey, Princeton University Press, 2002.
24 Voir entre autres : Anne Cauquelin «Le paysage n'est pas un lieu ", Paysages sur Commande, Rennes, Le Triangle, 1990, p. 93 ; et Arnold Berleant, The Aesthetics of Environment, Philadelphie, Temple University Press, 1992, p. 10 et suiv.

25 Kenneth Robert Olwig, Landscape, Nature, and the Body Politic, p. 10.

26 Simon Schama, Landscape and Memory.

27 Augustin Berque, Écoumène, Introduction à l'étude des milieux humains, Paris, Berlin, 2000, p. 13.

28 En français, le mot paysage apparaît au XVIe siècle. Catherine Franceschi, «Du mot paysage et de ses équivalents dans cinq langues européennes ", dans Michel Collot (dir.), Les Enjeux du paysage, Bruxelles, Ousia, 1997, p. 75-111.

29 Arnold Berleant, The Aesthetics of Environment, p. 1-13.

30 Jacques Aumont, "Le point de vue ", Communications, no. 38, 1983, p. 3-29; Jonathan Crary, Techniques of the Observer, MIT Press, Cambridge, Massachusetts, London, England (1990), 1995, p. 24-66.

31 T. J. Clark, The Painting of Modern Life: Paris in the Art of Manet and His Followers, Princeton, New Jersey, Princeton University Press (1984), 1999, p. 147-205.

32 L'environnement est la nature dont nous faisons l'expérience : la nature vécue (traduction de l'auteure). Arnold Berleant, op. cit., p. 10.

33 Glenlandia est une commande de la Horsecross for Threshold Artspace qui visait à l'origine à souligner de l'ouverture du Perth's Concert Hall.

34 Pour une description détaillée du projet, consulter le site : http://www.susan-collins.net. Voir également l'entrevue de Carlo Zanni, "Conversation with Susan Collins ", Magazine électronique du CIAC/CIAC's Electronic Magazine, no. 25, 2006, disponible à l'adresse suivante : http://www.ciac.ca/magazine/archives/no_25/ entrevue.htm.

35 Les effets dus au hasard, à la surprise et l'introduction d'éléments anecdotiques sont des aspects essentiels et récurrents dans le travail de Susan Collins. On les retrouve dans plusieurs autres projets antérieurs à Glenlandia, tels que : Woolwich Foot Tunnel, 1993, Pedestrian Gesture, 1994, In Conversation, 1997-1998. Voir, Susan Alexis Collins, Inhabited Content: An exploration into the role of the viewer through the realisation of In Conversation and other works, Ph.D. thesis, Fine Arts Department, University of Reading, 2001.

36 Hubert Damish, Théorie du nuage. Pour une histoire de la peinture, Paris, Éditions du Seuil, 1972, p. 38-39.

37 Ibid., p. 258.

38 Tiffany Holmes, « Environmental Awareness Through Eco-Visualization: Combining Art and Technology to Promote Sustainability ", Reconstruction: Studies in Contemporary Culture, 6.3, été 2006, http://reconstruction.eserver.org/063/holmes.html.

39 Propos de l'artiste, http://www.onetrees.org (consulté le 10 décembre 2007). 
40 Luther Burbank au début du siècle en raison de la vigueur particulière d'une variété de noyer. Le Paradox Vlach est une variété dérivée du Paradox et obtenue à partir d'un croisement avec un noyer noir non-identifié. Cet arbre fut d'abord planté par Jake Cover en 1904 sur la propriété d'un dénommé Vlach.

41 Pour plus de détails sur la description de ces expositions et le développement récent du projet, on peut consulter les sites : http:// www.onetrees.org et http://www.nyu.edu/projects/xdesign/onetrees/description/index.html.

42 Propos de l'artiste, http://www.onetrees.org (consulté le 10 décembre 2007).

43 Le Corbusier dans Françoise Choay, L'Urbanisme, utopies et réalités. Une anthologie, Paris, Seuil, 1965, p. 239.

44 Sur la base des notions de différence et de répétition, Ernestine Daubier discute le paradoxe à l'œuvre dans One Tree(s). Ernestine Daubier, « Les clones et les robots de Natalie Jeremijenko. La répétition/différence et autres stratégies subversives de représentation ", Parachute, no. 112, oct., nov., déc. 2003, p. 92-108.

45 Julia Kristeva, Pouvoirs de l'horreur, Paris, Seuil, 1980, p. 91.

46 Pour une description du projet, voir : Marc Pesce, The Playful World : How Technology is Transforming Our Imagination, New York, Ballantine Books, 2000, p. 220-30.

47 Pour une étude sociologique concernant les impacts de Tele-Garden sur les participants, on peut consulter : Margaret L. McLaughlin, Kerry K. Osborne et Nicole B. Ellison, «Virtual Community in a Telepresence Environment », dans S. Jones (éd.), Virtual Culture, London, Sage Publication, 1997, p. 146-68.

48 Edmond Couchot et Norbert Hillaire, L'Art numérique, Paris, Flammarion, 2003, p. 64-66.

49 Couchot a d'abord développé cette idée dans sa communication intitulée "De la communication à la commutation ", lors du colloque international Art média VIII, De l'esthétique de la communication au net art, organisé par Fred Forest et Annik Bureaud en partenariat avec l'Université de Salerne, le Centre français du commerce extérieur, l'École nationale supérieure, le GRIP et Leonardo/Olats, tenu à Paris, du 29 novembre au 2 décembre 2002.

50 Olivier Grau, Virtual Art: From Illusion to Immersion, Cambridge, Massachusetts, London, UK, MIT Press, 2003, p. 278.

51 Ibid., p. 69.

52 Ibid., p. 33-34.

53 Edmond Couchot et Norbert Hillaire, op. cit., p. 17.

54 Anne Cauquelin, "L'interface », op. cit., p. 233.

55 Entre autres : Mori (1999), Ouija (2000).

56 Kenneth Goldberg, "Virtual Reality in the Age of Telepresence ", Convergence Journal, vol. 4, no. 1, printemps 1998, p. 33-37.

57 Jacques Rancière, "Le partage du sensible ", entrevue menée par Christine Palmiéri, Etc. Montréal, sept., oct., nov. 2002, p. 34-41.

58 On peut voir des images tirées de Osmose sur le site de Char Davies : http://www.immersence.com.
59 Laurie McRobert, Char Davies' Immersive Virtual Art and the Essence of Spatiality, Toronto, Buffalo, London, University of Toronto Press, 2007, p. 13.

60 Char Davies, "Osmose: Notes on Being in Immersive Virtual Space», dans Colin Beardon et Lone Malmborg (éd.), Digital Creativity: a Reader, Lise, The Netherlands, Swets and Zeitlinger Publishers, 2002, p. 101-10. Disponible en format électronique: http://www. immersence.com/publications/char/2002-CD-Digital_Creativity. html (consulté le 12/10/2007).

61 Erkki Huhtamo, "Twin-Touch-Test-Redux : Media Archeological Approach to Art, Interactivity, and Tactility», dans Grau, Olivier (éd.), Media Art Histories, Cambridge, Massachusetts, London, UK, MIT Press, 2007, p. 71-101.

62 Louise Poissant (dir.), Esthétiques des arts médiatiques. Interfaces et sensorialités, C.I.E.R.E.C. Publications de l'Université de SaintÉtienne, Sainte-Foy, Presses de l'Université du Québec, 2003, p. 9.

63 Alain-Renaud Alain, "Interfaces et sensorialités ", dans Louise Poissant (dir.), op. cit., p. 87.

64 Laura U. Marks, Touch : Sensuous Theory and Multisensory Media, Minneapolis and London, University of Minnesota Press, 2002.

65 Derrick de Kerckhove, "Culture et médias numériques. Les médias et l'architecture de l'intelligence ", dans Louise Poissant, $o p$. cit., p. 59.

66 Philippe Poullaouec-Gonidec, "La condition paysagère du territoire ", dans Territoires. Le Québec, habitat, ressources et imaginaire, Québec, Musée de la civilisation, 2007, p. 19.

67 Anne Cauquelin, "L'interface. Le passage d'une philosophie du goût à une philosophie de l'action ", entrevue menée par Louise Poissant en mars 2002, dans Louise Poissant, op. cit., p. 229-37.

68 Voir particulièrement : Interactive Plant Growing, Trans Plant de Christa Sommerer et Laurent Mignonneau, Ozone de Stephan Barron et Paysage no 1 de Luc Courchesne.

69 Anne Cauquelin, 2002, op. cit., p. 196. 